\title{
Research on PLC Control Method of Industrial Field Based on Modbus Protocol
}

\author{
Wu Yuanxiu*, Wang Haiyan, Qin Zhenlong \\ Department of Mechanical and Electrical Engineering, Laiwu Vocational and Technical College, Jinan, \\ Shandong, 271100, China \\ *corresponding author
}

Keywords: Modbus Protocol, PLC, DCS, Automatic Water Supply, Monitoring System

\begin{abstract}
The existing single-chip microcomputer control system and control system have the disadvantage of high failure rate. Modbus protocol is recommended based on the use of PLC (programmable controller) and camera DCS (distributed control system). The implementation indicates that the established DCS system is stable, reliable and has low failure rate. Therefore, the communication between the online PLC and the advanced computer can be carried out. It also has the functions of remote flow monitoring, remote intelligent meter reading, remote switch control, etc.
\end{abstract}

\section{Introduction}

At present, most of China's water systems use DCS system, using semi manual and semi intelligent methods to ensure the safe and effective operation of the pipeline network. Among them, the current water supply control system mainly relies on a single chip microcomputer control system which is used for the physical relay of magnetic field side control[1]. However, the environment of industrial water plant is often very bad, and the damage of relay mechanical contact and control line is also relatively fragile, which has been in such a bad environment for a long time. Due to the influence of RS 485 communication protocol, this kind of damage often leads to the paralysis of the whole system. In addition, there are many tap water supply nodes in the industrial water supply system. Therefore, the single chip computer control system has the disadvantage of high failure rate. At present, the system process, pressure, water level, water temperature and other field competitions not only realize the collection of parameters, data storage, data statistics, data transmission, and human beings in order to achieve the interaction between computers, in addition, water is fully automated, semi-automatic transformation efforts[2]. In order to solve the problems of contact wear and line aging, the existing single chip microcomputer control system is transformed by PLC, and the physical relay in the existing control is replaced by the software relay in PLC. Compared with the problem that the whole system is paralyzed due to the existing RS 485 communication failure, the establishment of DCS water supply control system based on Modbus protocol corresponds to the problem that each control terminal is independent and does not affect each other. The modified water supply control system proves that the failure rate is low and the automation degree is high, which realizes the remote online monitoring of the automatic water supply system.

\section{Remote Control Structure of DCS Automatic Water Supply System}

The sensors, PLCs and actuators are connected via a fieldbus. This works in the field side of the whole system, and the working environment is very strict[3]. The required equipment includes waterproof, high temperature resistance, continuous operation, high voltage resistance, etc. The remote transceiver device is used to receive commands from the management layer. Generally speaking, the wireless GSM / GPRS communication network is used to execute the upper computer in the scene. At the end of the control, the remote transceiver device writes the received command to the PLC, which controls the actuator to control the command sent by the field layer device. For customer managers, it is convenient to collect the whole pipeline, view the defect warning, 
summary, release and manage the instructions sent on site.

PLC has high reliability and easy operation. In order to facilitate the field control and reliable operation, PLC is selected as the field controller. The DCS water supply control system has better stability and can operate reliably[4]. Two Modbus communication protocols in the camera DCS system Modbus communication protocol are applied in the industry standard protocol. It is possible that different electronic control devices can communicate with each other. Therefore, according to different manufacturers, the control devices are manufactured to wait for the industrial control network controlled by the camera DCS to be connected. The mod bus protocol does not specify the physical layer of the 7-tier structure of the OSI model. In order to facilitate the communication between networks, only message structure and response mechanism are defined.

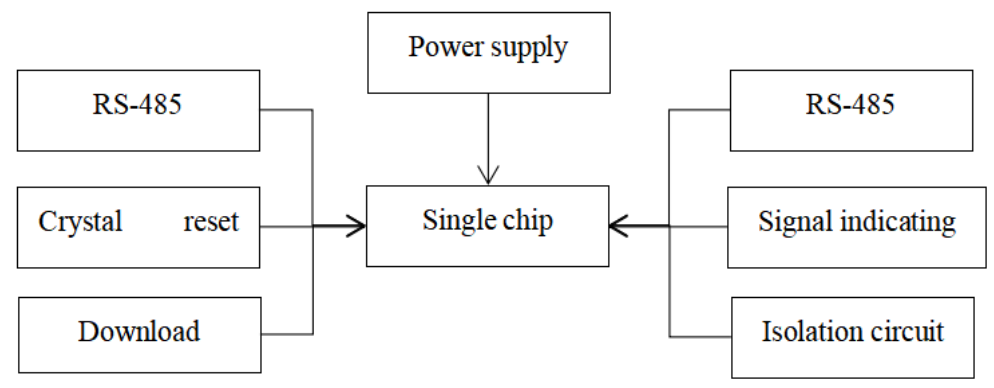

Figure 1 Hardware circuit model

Modbus protocol generally uses master-slave communication mode. As shown in the figure, the master-slave communication method of Modbus protocol is shown. When the host sends a data request command, multiple slave devices connected to the DCS network can receive messages. However, the slave needs to confirm the validity of the address. If the address is correct, the slave can send the requested data to the master in response to the command[5]. At this time, master-slave communication can be realized. Modbus communication protocol provides two message formats, ASCII and RTU. When communicating, it is necessary to select the correct channel between devices and set a consistent rate to realize the communication between devices. In addition to defining character formats, the Modbus protocol also requires data validation. The correct verification method in communication ensures the legitimacy of information transmission.

\section{DCS Automatic Water Supply System Based on PLC}

The control core of DCS automatic water supply system is PLC. PLC is very reliable and easy to operate. Compared with the previous control system, DCS system based on PLC has the advantages of flexible configuration, low cost and good stability. The Modbus protocol described by PLC in this report collects data through the sensor set in the pipeline network, and automatically provides remote real-time monitoring for the system, in order to realize GSM / GPRS wireless network and communication. During the remote monitoring process, data collection and status changes can be performed on the field side[6]. The software relay of PLC, the control of quantity design and power loss, secondly, the start and stop action of electric valve and deepwater pump on the water supply website, change, or water flow, pipeline pressure, in order to collect and control the water level signal of reservoir, or the communication control communication module between terminals and the PLC of monitoring center, the camera DCS system, use CPU 224 series. This machine integrates analog and digital input and output, has RS 485 programming port, supports a / D module expansion, and can communicate with the main computer and sensors. Monitor PLC.

\subsection{Flow Monitoring Module}

The remote automatic water supply system can realize the real-time monitoring of the water supply process of each unit, calculate the water consumption of the enterprise, and monitor the peak 
water free[7]. The $\mathrm{ZrN}$ - 100 ultrasonic flowmeter is used. The ultrasonic flowmeter has the functions of existing water flow statistics, wireless communication device, manual opening and closing device and back check valve, which can monitor the flow of pipeline automatically in real time. The software installed on the host can refresh and collect the remote flow in real time in different time periods every day, and analyze the trend statistics of the instantaneous flow collected by PLC according to different situations. The flow and pressure of water supply avoid the problem of small water intake or water supply. The ultrasonic flowmeter can collect the instantaneous flow of the pipeline and integrate the instantaneous flow to obtain the accumulated flow.

\subsection{Analog Quantity Conversion Module}

Because the computer can only process the digital quantity, and the information collected by the sensor on the device side is usually analog, so it is necessary to convert the analog quantity into the digital quantity that can be processed by the computer. In the industry, there are many things to convert the physical quantity of $4 \sim 20 \mathrm{~mA}$ current, or $\sim 10 \mathrm{~V}$ voltage analog quantity of the collection device, so it is necessary to convert the current value of $4 \sim 20 \mathrm{~mA}$ or $\sim 10 \mathrm{~V}$ voltage analog quantity to the corresponding digital quantity of the host processing. $4 \sim 20 \mathrm{~mA}$ current signal has strong interference and is easy to transmit[8]. Therefore, in the process of equipment acquisition, the physical quantity, node pressure and reservoir water level of water supply pipeline flow are converted into $4 \sim 20 \mathrm{~mA}$ current output. Then, a / D is converted into corresponding digital quantity, which is stored in PLC built-in register of host computer and discussed with realtime trend chart.

\subsection{MODBUS Acquisition Module}

The DCS water supply station supports Modbus protocol, uses equipment for collecting various monitoring parameters in the water supply network, and sets the corresponding RTU communication mode and the same serial port parameters to communicate with PLC. PLC uses ultrasonic flowmeter to collect the instantaneous flow of the pipeline, and calculates the total water consumption of the pipeline by ton. The collected information is converted by Modbus protocol and stored in the PLC memory used by the host computer. Through Modbus protocol, PLC can send the collected state information, such as voltage topology dislocation or current exceeding the standard, whether the power failure of water supply system is determined, water level of reservoir, start and stop of pump.

The use of meters supporting Modbus protocol allows information transmission between different electronic meters, which lays a foundation for master-slave communication method of DCS system. When the collected data is compared with the data written to PLC, when the host computer is outside the normal value, the host computer will give an alarm or automatically stop the pump to ensure the safety of the water supply system. When collecting information on the equipment side, the PLC on the field control side needs to give instructions. The program of PLC is designed in the form of LADA diagram and programmed in PLC.

\subsection{Execution Module}

Nodes of DCS control system can be connected to multiple sensors. During the driving of the camera DCS water supply system, the PLC is controlled by the instantaneous flow of the executive module, the collection and control of the reservoir water level, pressure pipeline network and other data, or the electric valve, the first level liquid pump, the deep-water well pump, etc[9]. At the same time, the end of website management requires the manager to execute the manager's commands according to the instructions of the customer center. The execution module uses PLC to process the parameters collected by the field equipment in a predetermined period of time, and logically determines the original parameters programmed in PLC after PLC processing and calculation. In case of failure, PLC module controls the completion of pump start and stop, electrical valve replacement and other operations, and feeds back the completed position conditions to PLC. At the same time, in case of any abnormal situation, PLC communicates with the main computer, the manager executes the instructions through the actuator, and the control of the terminal equipment 
ends.

\section{Remote Online Monitoring Software}

According to Modbus protocol and PLC control, the man-machine interface of DCS system is designed and described. Through Modbus communication protocol and PLC control, the running state of water supply system can be monitored online in real time. After the normal operation of the system, it means that the operator operates the online monitoring software of the host computer, queries the field data, rewrites the corresponding status register parameters in PLC, and realizes the online control function of the host computer.

Remote online monitoring software is the remote manual and automatic control mode of water supply system converted to realize serial communication. In order to rewrite through PLC control status register, MODBUS protocol indicates that "5" instruction code can be used. When the manual control mode is selected, the automatic control mode compulsorily inputs the exchange value of a single device through Modbus protocol and fails. Then, in order to achieve the start and stop of the corresponding components, in order to control the change, the automatic control mode is selected, the manual control mode is invalid, the data extraction method, time, power source state, discharge pipe network pressure, reservoir water level and other factors in the PLC operation parameter register are logically judged, and the corresponding start or stop is from Dynamic control equipment.

\section{Conclusion}

In order to transform the existing water supply control system, the communication between PLC and field sensor and the monitoring software of upper computer can be realized by using PLC based on Modbus protocol. The converted automatic water supply system has the functions of remote meter reading, analog quantity conversion, remote intelligent meter reading, remote online manual or automatic monitoring. Remote guide and automatic control mode conversion are realized by self described monitoring software of upper computer. Water supply time, power status, pipe network pressure and reservoir water level shall be judged logically, and the start and stop of corresponding equipment shall be controlled automatically. The application of automatic water supply control system can not only reduce the failure rate of equipment operation and labor cost of equipment maintenance, but also realize the automation of control engineering and improve production efficiency.

\section{Acknowledgements}

2019 Shandong vocational education reform project "reform and practice of hybrid teaching mode" programmable controller application technology "based on" Internet + "(C18G151107).

\section{References}

[1] ESA AHMED TORBOSH AL-QADASI. The Design and Research of Polyurethane Reactor Monitoring System Based on Kingview and PLC, 2017.

[2] Dong-Seong Kim, Hoa Tran-Dang. Distributed Control System for Ship Engines Using Dual Fieldbus: From Wired Technologies to Cloud Computing and the Internet of Things. Industrial Sensors and Controls in Communication Networks, 2019.

[3] Dixit S A, Jain A. Implementation of PPC-SSR as final control element and interfacing of PLC with LabVIEW using Modbus in two tank non interacting level control system, 2017.

[4] Nardone R, Rodriguez R J, Marrone S. Formal security assessment of Modbus protocol, 2017.

[5] A. Kozyra, K. Skrzypczyk, K. Stebel,. Remote controlled water craft for water measurement. Measurement, no. 111, pp. 105-113, 2017. 
[6] World Health Organization. Progress on drinking water sanitation and hygiene: 2017 update and SDG baselines. Geneva, World Health Organization [WHO], 2017.

[7] Hua Wang, Yijun Zhao, Fengnian Zhou,. Suspended sediment distribution under varied currents in the largest river-connected lake of China. Water Science \& Technology A Journal of the International Association on Water Pollution Research, vol. 18, no. 3, pp. ws2017167, 2017.

[8] Olson D. A DECLINING WATER SUPPLY: HOW UTAH CAN BECOME ADEPT AT ADAPTING TO THE IMPACTS OF CLIMATE CHANGE, 2017.

[9] Chih-Ta Lin, Sung-Lin Wu, Mei-Lin Lee. Cyber attack and defense on industry control systems. 2017 IEEE Conference on Dependable and Secure Computing. IEEE, 2017. 